\title{
Evaluación de la posibilidad de almacenamiento de energía renovable en el subsuelo de la cuenca Vasco-Cantábrica: estudio geomecánico de la Formación Keuper
}

\section{Assessment of the possibility of storing compressed air in the subsurface of the Basque-Cantabrian basin (North Spain): geomechanical study of the Keuper formation}

\author{
C. Laín ${ }^{1}$, B. Llamas ${ }^{1}$, R. Laín ${ }^{1}$, A.B. Sanchez ${ }^{1,2}$, M. Arlandi ${ }^{2}$ \\ ${ }^{1}$ ETSI Minas y Energía. Universidad Politécnica de Madrid. Alenza 4. 28003 Madrid. Spain. Email: bernardo.llamas@ \\ upm.es, ORCID ID: https://orcid.org/0000-0002-0513-672X, https://orcid.org/0000-0003-4755-5997, https://orcid. \\ org/0000-0003-4705-5546, https://orcid.org/0000-0002-6494-5404 \\ ${ }^{2}$ Túneles y Geomecánica SL. C/Alfonso Gómez 17, $3^{\circ}$ Local 11. 28037. Madrid. Spain. ORCID ID: https://orcid. \\ org/0000-0001-7935-671X
}

\section{RESUMEN}

El aumento de las energías renovables, como fuente de generación de electricidad, es una solución tecnológica respetuosa con el medioambiente y competitiva. Sin embargo, la garantía de suministro requiere la consideración de almacenes de energía para este tipo de soluciones cuya energía primaria es intermitente.

La tecnología de almacenamiento de aire comprimido en el subsuelo, supone una solución con gran capacidad de almacenamiento y gestión, además de que sus costes son los menores frente a otras soluciones. Sin embargo, requiere de una cuidadosa caracterización del macizo objetivo, con el fin de minimizar el riesgo exploratorio.

En este caso se evalúan las propiedades mecánicas de la formación Keuper, como formación donde construir la infraestructura energética propuesta, considerando para ello el concepto mini-CAES, como concepto de almacenamiento de aire comprimido en el subsuelo mediante cavidades someras. Se han llevado a cabo estudios mediante ensayos uniaxiales y propagación de onda con el fin de determinar los principales parámetros y de esta forma un coeficiente de seguridad.

De acuerdo al cálculo del coeficiente de seguridad, se considera que estas cavidades someras ofrecen un valor netamente superior frente a las cavidades convencionales, cuya profundidad es superior a los $700 \mathrm{~m}$. De esta forma se avanza en la definición de estas cavidades de menor capacidad volumétrica y profundidad.

Palabras clave: almacenamiento de Energía; caracterización geomecánica; sales; cuenca Vasco-Cantábrica.

\section{ABSTRACT}

The growth in renewable energies, as a source for electricity generation, is a technological solution environmentally friendly and competitive. However, to guarantee the supply requires considering the use of energy storage for such type of solutions whose primary energy is intermittent.

Recibido el 15 de enero de 2018 / Aceptado el 3 de mayo de 2018 / Publicado online el 1 de julio de 2018

Citation / Cómo citar este artículo: Laín C. et al. (2018). Evaluación de la posibilidad de almacenamiento de energía renovable en el subsuelo de la cuenca Vasco-Cantábrica: estudio geomecánico de la Formación Keuper. Estudios Geológicos 74(1): e78. https://doi. org/10.3989/egeol.43115.480

Copyright: () 2018 CSIC. This is an open-access article distributed under the terms of the Creative Commons Attribution-Non 4.0 International License. 


\begin{abstract}
The technology of storage of compressed air in the subsurface, supposes a solution with great storage capacity and management, besides that its costs are the least compared to other solutions. However, it requires a careful characterization of the target mass, in order to minimize the exploratory risk.

In this case, the mechanical properties of the Keuper formation are evaluated, as a geological formation to build the proposed energy infrastructure, considering the mini-CAES concept, as a concept of compressed air storage in the subsurface by means of shallow and small cavities. Studies have been carried out using uniaxial tests and wave propagation in order to determine the main parameters and thus a safety coefficient.

According to the calculation of the safety coefficient, it is considered that these shallow cavities offer a clearly superior value compared to conventional cavities, whose depth is greater than $700 \mathrm{~m}$. In this way, progress is made in the definition of these cavities of lower volumetric capacity and depth.
\end{abstract}

Keywords: Energy Storage; Geomechanical characterization; salt; Basque-Cantabrian basin.

\section{Introducción}

Entre las opciones consideradas para alcanzar un sector eléctrico bajo en emisiones de carbono, destaca la mejora de la eficiencia, el desarrollo de la tecnología de captura y almacenamiento de $\mathrm{CO}_{2}$ y el fomento de las energías renovables (IPCC, 2014).

En el último caso, Europa en general está promoviendo la transformación del sector eléctrico apoyándose en las energías renovables (EUROSTAT, 2016). Destacando la energía solar que supuso el 5,5\% y la energía eólica, que alcanzó el 9\% del total de la electricidad consumida. Nuestro país cuenta con un significativo parque de energía renovable, destacando la energía eólica, que supuso el $23 \%$ de la energía consumida durante el año 2014.

Pero para la consolidación de las energías renovables, se hace necesario establecer tecnologías que permitan gestionar de una forma eficiente la energía (Kyriakopoulos et al., 2016; Madlener \& Latz, 2013; Lund \& Salgi, 2009). De esta forma se podrá minimizar la incertidumbre planteada entre la (i) previsión y realidad de la demanda de consumo y (ii) la previsión de la producción de energía en instalaciones de generación renovable y la realidad.

Para poder garantizar el suministro de energía, procedente de energías renovables intermitentes (solar y eólica) surgen las tecnologías de almacenamiento de energía (Ibrahim et al., 2008; Chen et al., 2009). Son pocas las alternativas consideradas a escala comercial para el almacenamiento de energía eléctrica con capacidad instalada superior a los $100 \mathrm{MW}$ : bombeo-almacenamiento de agua (PHS, por sus siglas en inglés) y almacenamiento de aire comprimido en el subsuelo (CAES, por sus siglas en inglés) (Chen et al., 2009). Ambas tecnologías permiten el almacenamiento y gestión de significativa cantidad de energía (con una potencia instalada superior a los
100 MW) (Lund \& Salgi, 2009) y se encuentran en fase industrial con diferentes referencias a nivel europeo e internacional (Luo et al., 2015).

El almacenamiento de aire comprimido en el subsuelo supone a priori un menor coste de inversión y operación (Luo \& Wang, 2013; Liang et al., 2016), pero el elevado riesgo derivado de la exploración del subsuelo dificulta el desarrollo de la misma. Entre las opciones consideradas para llevar a cabo el almacenamiento de energía en el subsuelo, se consideran las cavidades salinas (McCartney et al., 2016) o las cavidades excavadas en roca con recubrimiento de hormigón (Kim et al., 2012) o la inyección en acuíferos (Fertig y Apt, 2011). De entre las opciones consideradas, la utilización de cavidades salinas ofrece las mejores características desde el punto de vista técnico-económico (Liang et al., 2016; Bozzolani, 2010). También la eficiencia energética en la tecnología CAES convencional es sensiblemente inferior a la de bombeo de agua, y es por ello que se desarrollan nuevos conceptos como el CAES-Adiabático (Llamas et al., 2017a).

La realización de cavidades someras como solución para el almacenamiento de energía ofrece adicionalmente al menor impacto ambiental - por su menor volumen de extracción de salmuera, menor coste de inversión (CAPEX) al reducir el volumen de cavidad y profundidad objetivo, así como un menor riesgo de exploración (Llamas et al., 2018).

\section{Contexto Geológico}

La Cuenca Vasco-Cantábrica se encuentra situada en el límite septentrional de la Península Ibérica, en el extremo occidental de la cadena montañosa de los Pirineos, y con una superficie de $25.000 \mathrm{~km}^{2}$. La evolución geológica de esta cuenca es el resultado de los cambios sedimentarios, 
tectónicos, paleogeográficos y paleoclimáticos desde finales del Paleozoico (U.T.E. Tecnología de la Naturaleza, 2012). Su configuración refleja la superposición de etapas compresivas alpinas a etapas distensivas mesozoicas (Vera, 2010). La exploración petrolífera ha confirmado la enorme potencia de la sedimentación, especialmente la sucesión Mesozoica (Carracedo-Sanchez et at, 2012): algunos de los sondeos más profundos en España fueron realizados en esta cuenca, con profundidades de 5.991 y $5.843 \mathrm{~m}$ (Castillo-5 y Urbasa respectivamente) (Lanaja \& Navarro, 1987).

Hacia el sur constituye una lámina cabalgante sobre las Cuencas Terciarias del Duero y del Ebro, evidenciado por el sondeo Astrain (Lanaja \& Navarro, 1987); mientras que hacia el norte se prolonga offshore hasta el Golfo de Vizcaya. El límite occidental está delimitado por el Macizo Paleozoico Asturiano y el oriental por los Macizos Paleozoicos Vascos y los Pirineos (Figura 1).

En el Triásico las cuencas sedimentarias con facies germánicas instaladas sobre la Placa Ibérica registraron varios episodios salinos (Vera, 2010). Las principales áreas que manifiestan la sedimentación salina triásica son: la Cuenca Vasco-Cantábrica, la cubeta de Ballobar (en la Depresión del Ebro), la cubeta del Maestrazgo, la Cuenca de Valencia-Cuenca y el amplio dominio Prebético-Subbético.

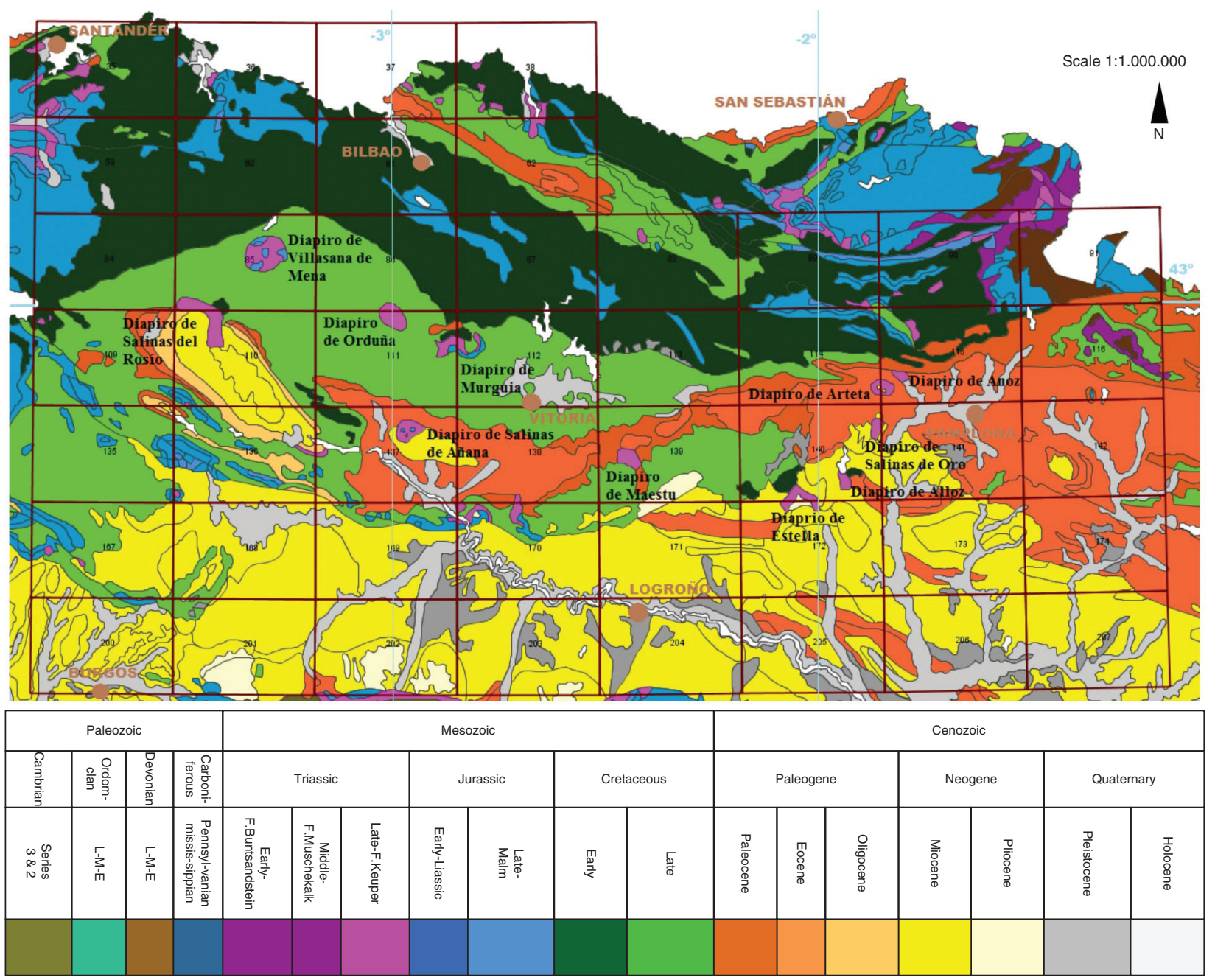

Fig. 1.-Contexto geológico de la zona en estudio. Detalle de los 11 diapiros identificados. 
Dentro del Triásico destacan los depósitos de facies Keuper, que forman la parte superior de la serie triásica, y están constituidos por lutitas de colores rojizos, azulados y verdes con intercalaciones de yeso y localmente con inclusiones de ofitas (Vera, 2010). Tanto en la base como especialmente en la parte superior de la sucesión predominan las lutitas de tonalidades verdosas y azuladas con intercalaciones calcáreas que forman secuencias de orden métrico formadas de base a techo por lutitas versicolores, dolomías oquerosas y dolomías laminadas.

Las estructuras diapíricas de materiales salinos Triásicos (Keuper) de la Cuenca Vasco-Cantábrica son características de la evolución de una cuenca con niveles salinos profundos, afectada primero por un periodo tensional y deformada después por uno compresional. El periodo tensional desencadenó la creación temprana y posterior evolución de los diapiros, mientras que el compresional provocó profundas modificaciones en su geometría original, además de una inversión estructural generalizada. Alguna de estas estructuras diapíricas son aflorantes y otras solo se reconocen mediante geofísica. Aquellas estructuras aflorantes tienen como ventaja fundamental la flexibilidad de desarrollar cavidades a diferentes profundidades, pudiendo de esta forma amoldarse al entorno energético - proximidad con parques eólicos - y de esta forma, ajustar la cavidad a la capacidad de almacenamiento del entorno (B. Llamas et al., 2017b).

\section{Materiales y métodos}

El objeto de la investigación se centra en la caracterización geomecánica de la halita del Keuper, mediante ensayos Uniaxiles y determinación de la presión máxima del macizo, que permitirá determinar la presión máxima de operación de una cavidad para la construcción de un almacenamiento de aire comprimido en el subsuelo. Para llevar a cabo el estudio, se utilizaron testigos procedentes de un sondeo mecánico a una profundidad suficiente como para considerar representativos los resultados (muestras tomadas a profundidad de $506 \mathrm{~m}$ ).

Adicionalmente, se considera el concepto de MINI-CAES (B. Llamas et al., 2018), o lo que es lo mismo: desarrollo de cavidades someras que, si bien acumulan una menor cantidad de energía, como ventajas se recoge un menor impacto medioambiental, menor riesgo exploratorio y menor coste de inversión.

El conocimiento del comportamiento mecánico de la sal y los agregados salinos se hace imprescindible debido a sus propiedades físicas como la baja permeabilidad, el comportamiento visco-plástico y también hay que tener en cuenta, que se trata de un material altamente deformable y reactivo. El comportamiento constitutivo de las sales se describe como una combinación de comportamiento elástico y fluencia - de acuerdo a la ley Norton, obligando a estudiar su comportamiento en el tiempo (Heijidra \& Prii, 1992).

\section{Descripción de los ensayos}

El material involucrado en la investigación se compone de 10 testigos de sal procedentes de la Cuenca Vasco-Cantábrica (Figura 2). Se trata de una sal masiva cristalina, con algunas intercalaciones esporádicas de arcillas y/o margas en forma de brechas. En general, se pueden diferenciar dos tramos:

- Uno inferior de sal cristalina, de tono gris claro o blanquecino, grandes cristales de halita y escaso contenido en insolubles. Frecuentemente presenta textura cataclástica, con fragmentos aplastados y orientados, con cierta orientación fluidal pero sin llegar a desarrollar textura milonitica. Es muy difícil poder distinguir planos de estratificación, ya que ha habido una recristalización generalizada, logrando reconocerse como mucho un cierto bandeado mal definido.

- Otro superior de sal masiva cristalina granulada de tono gris oscuro y algo de contenido en insolubles, pudiendo aparecer tramos brechoides de fragmentos de arcillas y margas grises. No se reconocen planos de estratificación, pero se puede llegar a observar un cierto bandeado por alternancia de tramos más oscuros y más claros. Hacia el techo de la unidad se hace más patente el bandeado, marcado por presencia de arcillas y capas de potasas.

Para llevar a cabo los ensayos uniaxiales de resistencia a compresión simple, se utiliza la normativa 


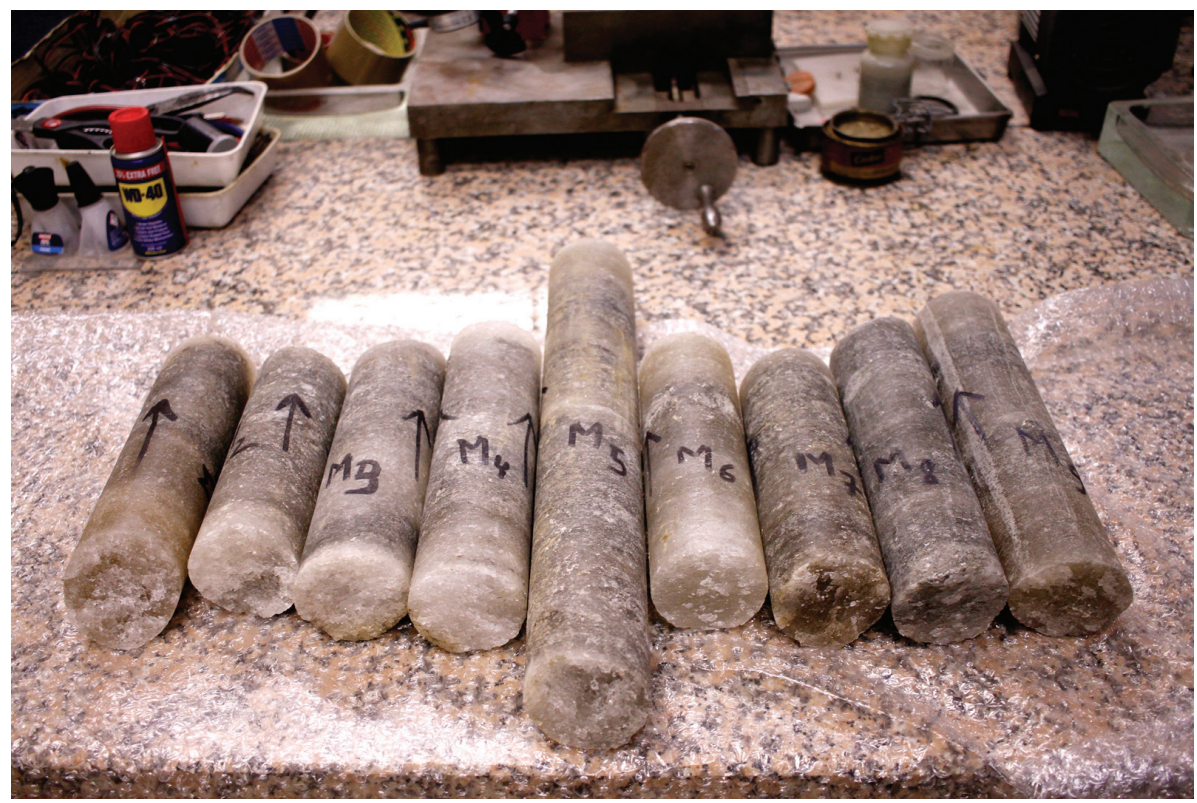

Fig. 2.-Muestras recibidas, antes de preparación de las mismas.

UNE 22-950-3:1990 y ASTM D 7012-94, mientras que, para la determinación de la velocidad de transmisión de ondas, se sigue la normativa ASTM D 2845-00.

\section{Módulos dinámicos}

Las ondas sísmicas producidas en la tierra dependen de la elasticidad de los materiales a través de los que se propagan. La velocidad de las ondas depende de las constantes elásticas de estos materiales. Las expresiones de las constantes elásticas en función de la velocidad de las ondas (Ecuación 1, Ecuación 2, Ecuación 3 y Ecuación 4) son las siguientes (King, 1983):

$$
\mu=\frac{\frac{V_{P}^{2}}{2 V_{S}^{2}}-1}{\frac{V_{P}^{2}}{V_{S}^{2}}-1}
$$

Ecuación 1. Coeficiente de Poisson dinámico

$$
E_{d}=\frac{\rho\left(3 V_{P}^{2}-4 V_{S}^{2}\right)}{\frac{V_{P}^{2}}{V_{S}^{2}}-1}=\frac{2 \rho V_{S}^{2}}{1+\mu}
$$

Ecuación 2. Módulo de Young dinámico.

$$
G_{d}=\rho V_{S}^{2}=\frac{E_{d}}{2(1+\mu)}
$$

Ecuación 3. Módulo de corte dinámico.

$$
B_{d}=\rho\left(V_{P}^{2}-\frac{4 V_{S}^{2}}{3}\right)=\frac{E_{d}}{3(1-2 \mu)}
$$

Ecuación 4. Módulo de compresibilidad dinámico. donde,

$$
\begin{array}{ll}
\mathrm{V}_{\mathrm{P}} & \begin{array}{l}
\text { es la velocidad real de las ondas } \mathrm{P} . \\
\mathrm{V}_{\mathrm{S}}
\end{array} \\
\rho & \begin{array}{l}
\text { es la velocidad real de las ondas } \mathrm{S} . \\
\text { es la densidad de la roca }
\end{array} \\
\mu & \begin{array}{l}
\text { es el coeficiente de Poisson diná- } \\
\text { mico de la roca }
\end{array} \\
\mathrm{E}_{\mathrm{d}} & \begin{array}{l}
\text { es el módulo de Young dinámico de } \\
\text { la roca }
\end{array} \\
\mathrm{G}_{\mathrm{d}} & \begin{array}{l}
\text { es el módulo de corte dinámico de la } \\
\text { roca }
\end{array} \\
\mathrm{B}_{\mathrm{d}} & \begin{array}{l}
\text { es el módulo de compresibilidad } \\
\text { dinámico de la roca }
\end{array}
\end{array}
$$

Para llevar a cabo este ensayo, se utiliza un conductivímetro y osciloscopio (figura 3), realizándose 


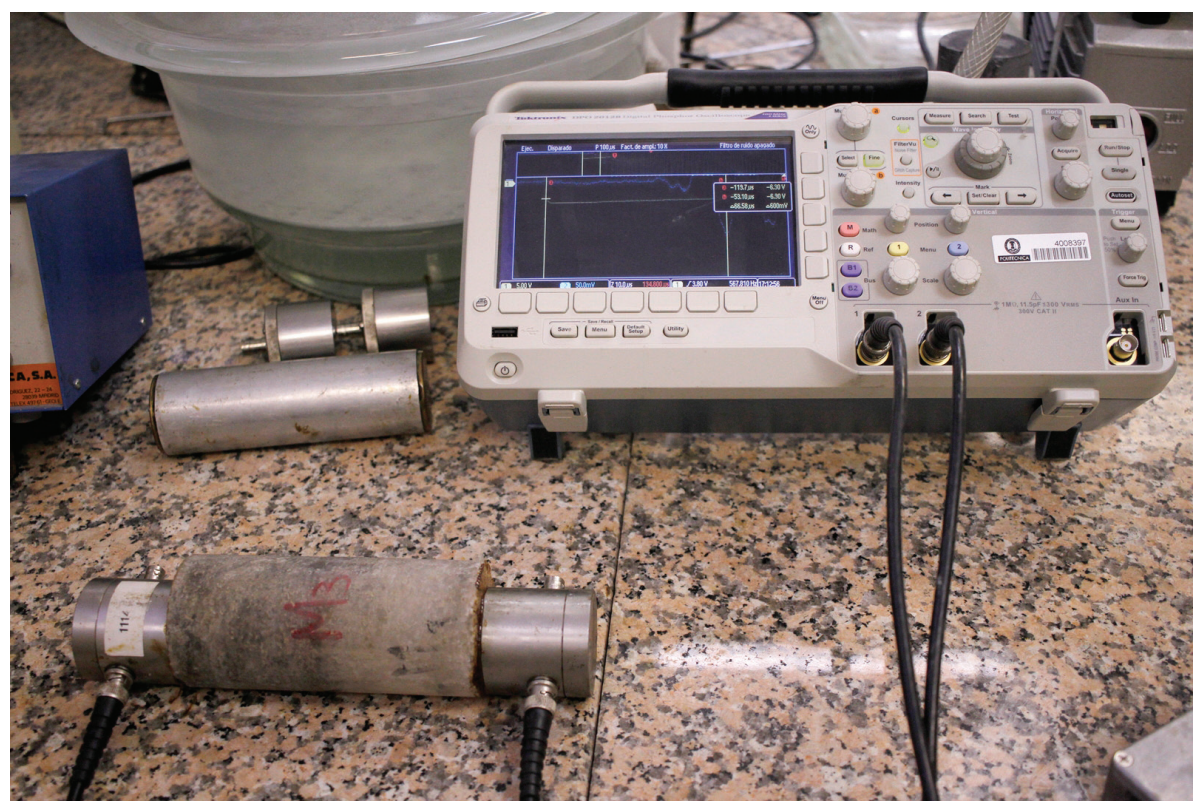

Fig. 3.-Determinación de la velocidad de propagación.

10 medidas para cada una de las variables en determinación.

El índice de continuidad (Ecuación 5) de una roca se calcula dividiendo el valor teórico de la velocidad que, de acuerdo con su composición mineralógica, deberían tener las ondas $\mathrm{P}$ por la velocidad real:

$$
I C=\frac{V_{p}}{V_{P . T .}} \cdot 100
$$

\section{Ecuación 5. Índice de continuidad de la roca.}

Donde, IC es el índice de continuidad, $\mathrm{V}_{\mathrm{P}}$ es la velocidad real de las ondas $\mathrm{P}$ y $\mathrm{V}_{\text {P.т. }}$ es la velocidad teórica de las ondas $\mathrm{P}$ según la composición mineralógica de la roca.

Cuanto mayor es la fracturación o la porosidad de una roca menor es su índice de continuidad, ya que la velocidad de las ondas $\mathrm{P}$ se ve afectada negativamente.

La velocidad teórica de propagación se calcula mediante la siguiente fórmula (Ecuación 6):

$$
\frac{1}{V_{P . T .}}=\sum \frac{C_{i}}{V_{p_{i}}}
$$

Ecuación 6. Velocidad teórica de propagación.
Donde, $\mathrm{C}_{\mathrm{i}}$ son las concentraciones en tanto por uno de los minerales contenidos en la roca y $\mathrm{V}_{\mathrm{Pi}}$ son las velocidades de las ondas $\mathrm{P}$ en cada uno de los minerales.

Este tipo de ensayo es no destructivo y su utilización en el presente trabajo permite establecer una primera valoración del testigo, con el fin de determinar si este está fracturado y por tanto afectará los resultados del ensayo de compresión simple.

\section{Ensayo de compresión simple}

Permite determinar la resistencia dela roca, siendo un parámetro importante en la clasificación de una roca desde el punto de vista mecánico y uno de los criterios de rotura más utilizados (Hoek, 1990; Hoek \& Brown 1980).

Este ensayo puede proporcionar también las constantes elásticas de la roca, es decir, su módulo de Young y su coeficiente de Poisson, para ello es necesario medir las deformaciones axiales y laterales de la probeta durante el proceso de carga, lo cual se realiza mediante cuatro bandas extensométricas, dos axiales y dos laterales, que se pegan directamente sobre la roca (figura 4). Los resultados de estos ensayos serán los considerados para la determinación del factor de seguridad. 


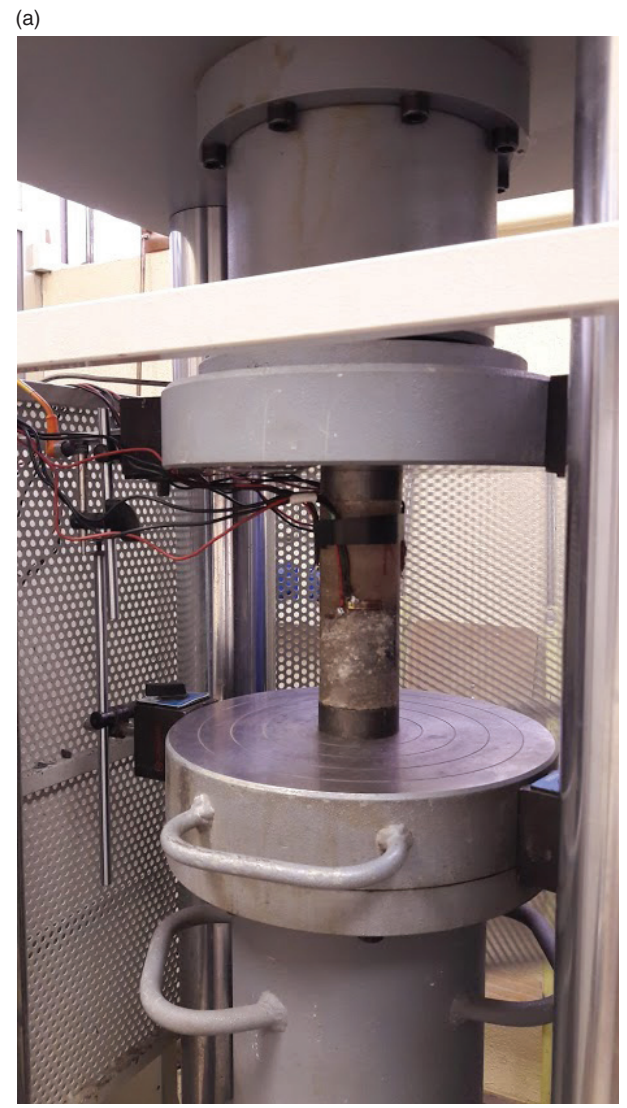

(b)

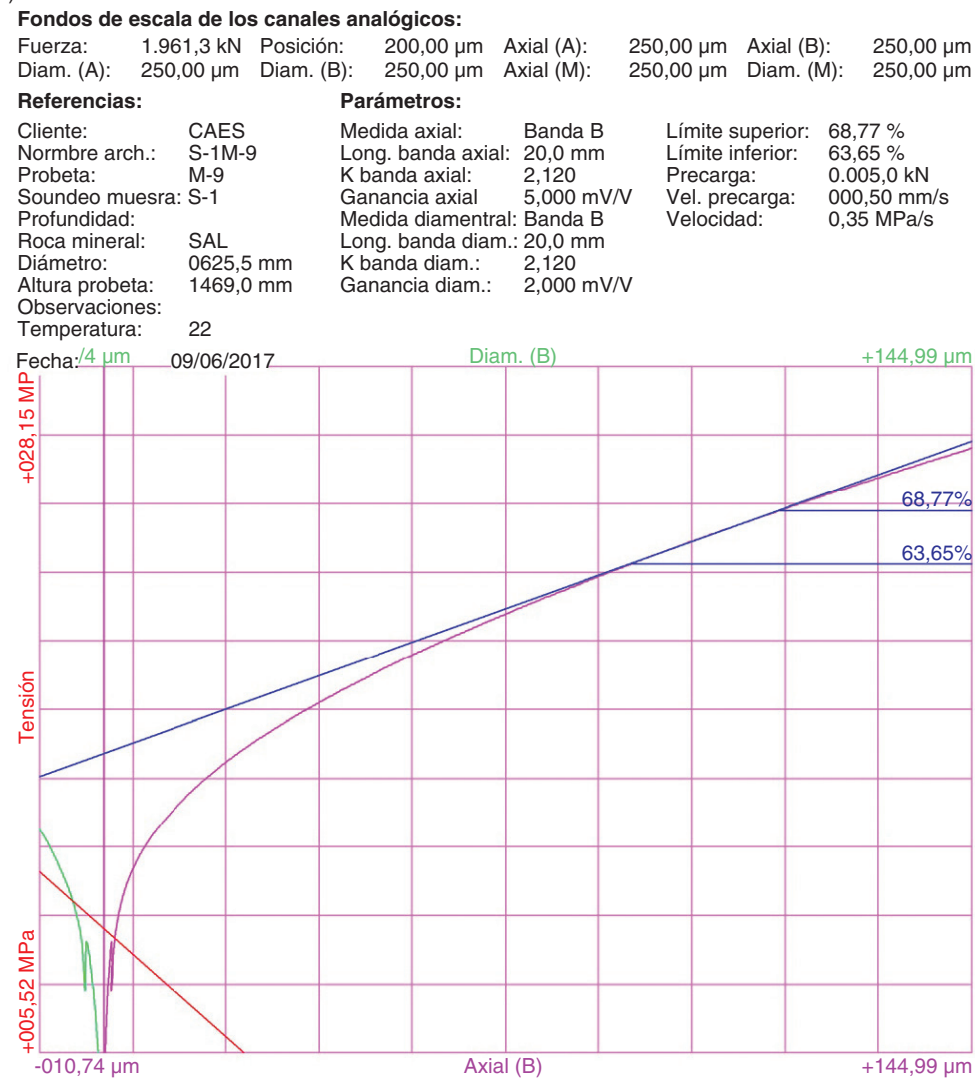

Fig. 4.-Ensayo uniaxal para la muestra M9.

\section{Coeficiente de seguridad de la cavidad}

La forma propuesta para determinar el coeficiente de seguridad, parte de la determinación de la presión máxima, que en este caso, viene dada por la Ecuación 7 (formulación basada en datos empíricos y experiencia del equipo de investigación) y Ecuación 8 :

$$
P_{\max }=0.18 \cdot h
$$

Ecuación 7. Presión máxima de operación en relación con la profundidad (h).

$$
P_{\max }=\rho_{\text {halita }} \cdot g \cdot h
$$

\section{Ecuación 8. Calculo de la presión litostática}

Donde $\mathrm{h}$ es la profundidad, $\rho_{\text {halita }}$ (teóricamente $\left.2,1 \mathrm{~g} / \mathrm{cm}^{3}\right)$, g la gravedad. De esta forma, se proponen dos formulaciones para la determinación de la presión máxima de trabajo de la cavidad propuesta.
Para los estudios de seguridad recogidos en el presente trabajo, se tomará el mínimo valor establecido por estas dos ecuaciones.

Para la determinación de la presión mínima de la cavidad (evitando de esta forma la convergencia de la cavidad) se establece igualmente mediante la Ecuación 9:

$$
P_{\min }=\frac{1}{2} P_{\max }
$$

Ecuación 9. Presión mínima de operación de la cavidad.

La tensión de rotura $\left(\mathrm{P}_{\text {rotura }}\right)$ de las muestras ensayadas ha alcanzado un promedio de $33,23 \mathrm{MPa}-$ valor máximo de 34,10 MPa y mínimo de $31,30 \mathrm{MPa}$. De esta forma, se puede determinar el coeficiente de seguridad de la infraestructura mediante la Ecuación 10. 


$$
\begin{aligned}
\text { (a) } \operatorname{coef}_{\text {Seguridad }} & =\frac{P_{\text {rotura }}}{P_{\text {litostatica }}} \\
\text { (b) } \operatorname{coef}_{\text {Seguridad }} & =\frac{P_{\text {rotura }}}{P_{\max }}
\end{aligned}
$$

Ecuación 10. Coeficiente de seguridad para diferentes profundidades; (a) considerando la presión litostática y (b) la presión máxima.

Se ofrecen en este cálculo dos ecuaciones, tomando el menor valor de las dos ecuaciones indicadas.

\section{Resultados}

La velocidad de propagación de las ondas $\mathrm{P}$ en las 10 muestras se aproxima de una forma razonable a la velocidad teórica de la halita $(4.500 \mathrm{~m} / \mathrm{s})$. En la figura 5 se recogen los valores obtenidos de las muestras P y S. La velocidad promedio de la primera de ellas es de $4.437 \mathrm{~m} / \mathrm{s}$, con una desviación estándar de 40,99 y un coeficiente de variación de $0,92 \%$. El valor promedio obtenido es un $1,4 \%$ inferior al valor teórico $(4.500 \mathrm{~m} / \mathrm{s})$, lo que denota que el macizo es compacto y sin apenas fracturación, lo cual lo hace muy favorable para la aplicación en estudio.
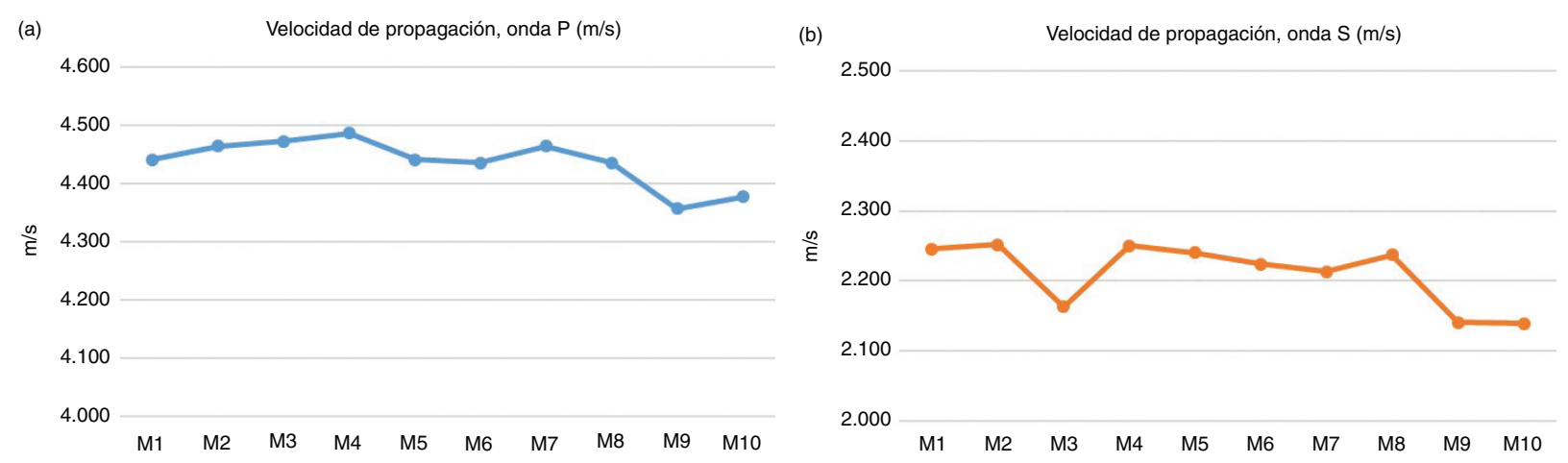

Fig. 5.-Velocidades de propagación para las diferentes muestras; (a) ondas P; (b) ondas S.

\begin{tabular}{|c|c|c|c|c|c|c|c|c|c|c|c|}
\hline & Unidades & $\begin{array}{l}\text { Número de } \\
\text { muestras (n) }\end{array}$ & $\begin{array}{l}\text { Máximo } \\
\text { (máx.) }\end{array}$ & $\begin{array}{l}\text { Mínimo } \\
\text { (mín.) }\end{array}$ & $\begin{array}{l}\text { Rango } \\
\text { (R) }\end{array}$ & $\begin{array}{c}\text { Rango } \\
\text { medio }\left(R_{m}\right)\end{array}$ & $\begin{array}{l}\text { Desviación } \\
\text { estándar } \\
(\sigma)\end{array}$ & $\begin{array}{c}\text { Varianza } \\
\qquad\left(\sigma^{2}\right)\end{array}$ & $\begin{array}{c}\text { Media } \\
\text { aritmética }\end{array}$ & $\begin{array}{c}\text { Media } \\
\text { geométrica } \\
(\mathrm{X})\end{array}$ & $\begin{array}{c}\text { Coeficiente } \\
\text { de variación } \\
\text { (Cv \%) }\end{array}$ \\
\hline$V_{P}$ & $\mathrm{~m} / \mathrm{s}$ & 10 & 4.486 & 4.357 & 129 & 64,49 & 41,02 & 1.683 & 4.437 & 4.437 & 0,92 \\
\hline$V_{S}$ & $\mathrm{~m} / \mathrm{s}$ & 10 & 2.252 & 2.139 & 113 & 56,35 & 45,31 & 2.053 & 2.211 & 2.210 & 2,05 \\
\hline
\end{tabular}

Tabla 1.-Datos estadísticos de los resultados obtenidos en la medición de las velocidades de propagación. 


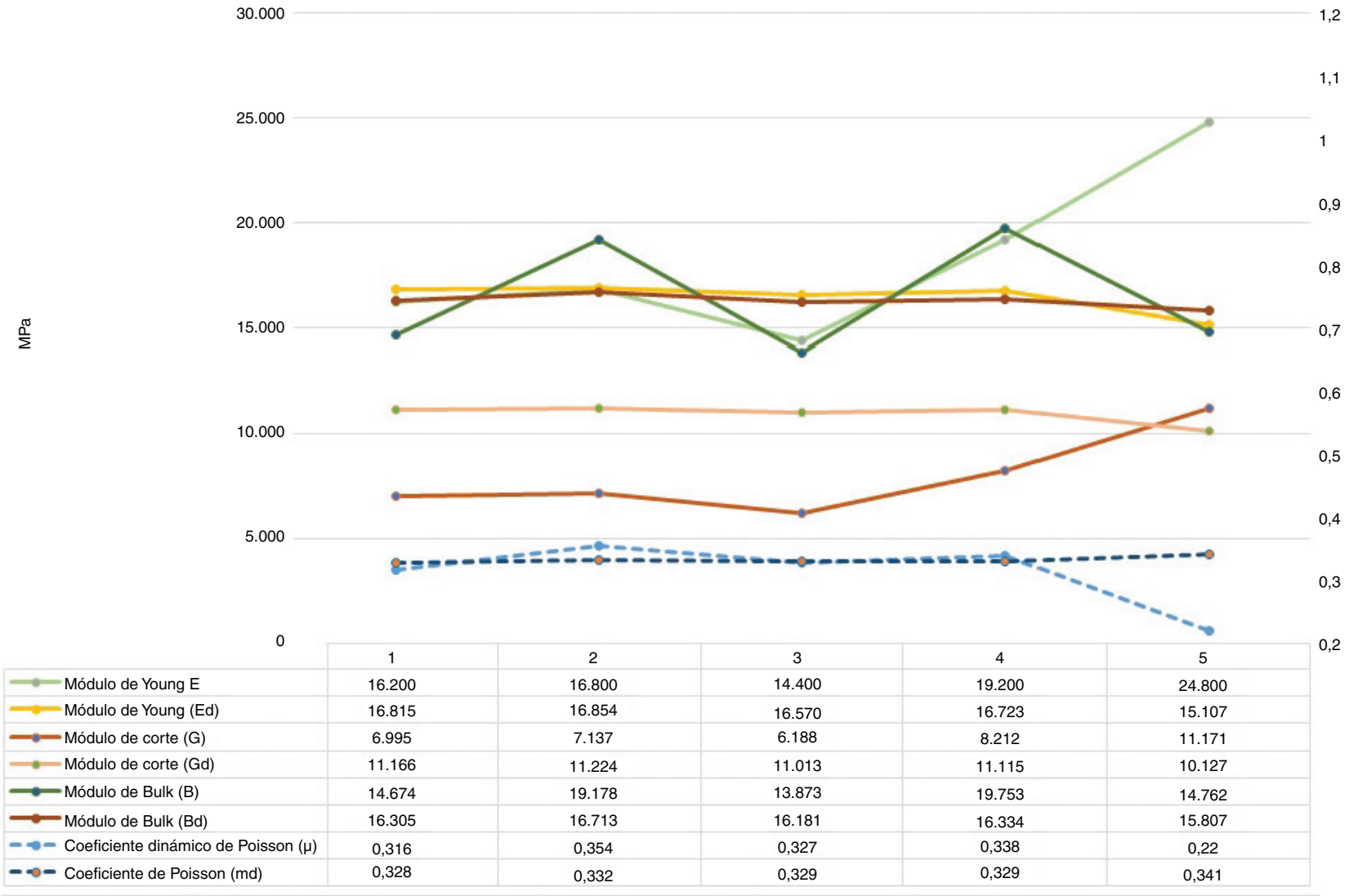

Fig. 6.- Resultados obtenidos en los ensayos de compresión simple y determinación de los módulos y coeficientes dinámicos.

Tabla 2.-Datos estadísticos de los resultados obtenidos en los ensayos uniaxiales.

\begin{tabular}{lrrrr}
\hline & \multicolumn{1}{c}{$\mu$} & \multicolumn{1}{c}{$\mathrm{E}$} & $\mathrm{G}$ & $\mathrm{B}$ \\
\hline Número de muestras $(\mathrm{n})$ & 4 & 4 & 4 & 4 \\
Maximo $(\mathrm{max})$ & 0,354 & 19.200 & 8.212 & 19.753 \\
Minimo $(\mathrm{min})$ & 0,316 & 14.400 & 6.188 & 13.873 \\
Rango $(\mathrm{R})$ & 0,038 & 4.800 & 2.024 & 5.880 \\
Rango medio $\left(\mathrm{R}_{\mathrm{m}}\right)$ & 0,019 & 2.400 & 1.012 & 2.940 \\
Desviación estándar $(\sigma)$ & 0,016 & 1.982 & 832 & 3.025 \\
Varianza $\left(\sigma^{2}\right)$ & 0,000263 & 3.930 .000 & 692.089 & 9.148 .414 \\
Media aritmética & 0,334 & 16.650 & 7.133 & 16.869 \\
Media geométrica $(\bar{X})$ & 0,333 & 16.562 & 72.963 & 183.685 \\
Coeficiente de variación $(\mathrm{C} v$ \%) & 4,86 & 11,91 & 11,66 & 17,93 \\
\hline
\end{tabular}

operación de una cavidad para almacenamiento de aire comprimido.

En la tabla 3 se refleja la presión litostática del macizo (Ecuación 8) y máxima de operación Ecuación 7) y el cálculo del coeficiente de seguridad considerando la tensión promedio de rotura obtenida en los ensayos uniaxiales llevados a cabo en el presente estudio. Siendo para los casos en estudio el coeficiente de seguridad superior a 1 , pero es tanto mayor cuanto menor es la profundidad (en el rango en estudio el coeficiente de seguridad calculado es más del doble en la menor profundidad considerada 
Tabla 3.-Determinación del coeficiente de seguridad de la infraestructura para diferentes profundidades consideradas.

\begin{tabular}{lcccr}
\hline & & \multicolumn{3}{c}{ Profundidad $(\mathrm{m})$} \\
\cline { 3 - 5 } & $\mathrm{MPa}$ & 6,18 & 10,30 & 14,00 \\
\hline $\mathrm{P}_{\text {litostática }}$ & - & 5,38 & 3,23 & 2,30 \\
Coef. Seguridad & $\mathrm{MPa}$ & 5,40 & 9,00 & 12,60 \\
& - & 6,15 & 3,69 & 2,64 \\
$\mathrm{P}_{\max }$ & $\mathrm{MPa}$ & 33,23 & & \\
Coef. Seguridad & & &
\end{tabular}

frente a la mayor). Estos resultados indican que el concepto de desarrollo de almacenamiento de energía, basado en menores cavidades y a menor profundidad son más seguras.

\section{Conclusiones}

El incremento de la energía renovable es una realidad, contribuyendo de esta forma en la descarbonización del sistema eléctrico y la lucha contra el cambio climático. Con el fin de incrementar la disponibilidad de fuentes renovables intermitentes, se propone la utilización de almacenes de energía en el subsuelo, mediante el desarrollo del concepto mini-CAES.

Las condiciones geomecánicas de un macizo salino son fundamentales para el desarrollo de cavidades cuyo fin es almacenar y gestionar energía, mediante la acumulación de aire comprimido en el subsuelo.

Mediante este estudio se han determinado los parámetros fundamentales desde el punto de vista geomecánico para la determinación de estabilidad de una cavidad y, limitar la convergencia de la misma con el fin de minimizar los efectos de subsidencia en superficie

En este caso, siendo las muestras procedentes de la cuenca Vasco-Cantábrica, se ha determinado que, mediante el estudio de las velocidades de propagación de las ondas $\mathrm{P}$ y S, el macizo en estudio presenta una baja fracturación y una resistencia a compresión elevada lo cual lo hace idóneo para el fin considerado. El índice de continuidad determinado para las muestras en estudio es cercano a 1 , lo cual incide en las condiciones geomecánicas favorables.

Se han establecido los valores de trabajo, con respecto a las presiones de operación en relación con la profundidad y determinado el coeficiente de seguridad. En todos los casos, el coeficiente de seguridad es superior a 1 , pero este coeficiente es tanto mayor cuanto menor es la profundidad de la infraestructura.

El trabajo aquí presentado, demuestra la idoneidad del concepto mini-CAES, como estructuras someras, ya que el estudio geomecánico realizado indica un mayor coeficiente de seguridad de cavidades someras frente a infraestructuras más profundas.

Estas cavidades son compatibles con los diapiros aflorantes en la cuenca Vasco-Cantábrica y, por tanto dicha región podría albergar esta nueva infraestructura energética, capaz de almacenar y gestionar energía renovable, gracias a los parques eólicos instalados en dicha región.

Próximos trabajos completarán el estudio mediante ensayos de deformación en el tiempo (ensayos de fluencia) y de fatiga, ya que se considera que el macizo estará sometido a presiones que excederán del límite elástico y, por tanto, estará sometido a una deformación constante. Diferentes ciclos de carga y descarga del macizo deberán ser estudiados con el fin de limitar dicha deformación siendo necesario trabajar dentro del régimen de fluencia secundario. Así mismo, se abordarán en los ensayos triaxiales. Todo ello, permitirá realizar un modelo y simulación numérica para establecer el comportamiento de la cavidad.

\section{Referencias}

Bozzolani, E. (2010). Techno-economic analysis of compressed air energy storage systems. Crandfield University, 203 pp. URI: http://dspace.lib.cranfield. ac.uk/handle/1826/6786

Carracedo-Sanchez, M.; Sarrionandia, F. \& Juteau, T. (2012). El Vulcanismo Submarino de Edad Cretácica de la Cuenca Vasco-Cantábrica. Revista de la Sociedad Española de Mineralogía, 16: 260-267.

Chen, H.; Cong, Y.; Yang, W.; Tan, C.; Li, Y. \& Ding, Y. (2009) Progress in electrical energy storage: a critical review. Progress in Natural Science, 19(3): 291-312. https://doi.org/10.1016/j.pnsc.2008.07.014

EUROSTAT. http://ec.europa.eu/eurostat/statistics-explained/index.php. Acceso el 27 de junio de 2016.

Fertig, E. \& Apt, J. (2011) Economics of compressed air energy storage to integrate wind power: a case study in ERCOT. Energy Policy, 39(5): 2330-2342. https:// doi.org/10.1016/j.enpol.2011.01.049

U.T.E. Tecnología de la Naturaleza SL \& Grama Estudio de Arquitectura y Medioambiente SL (2012). Síntesis Geológica de la Comunidad Autónoma del País Vasco. Departamento de Medioambiente y Política Territorial, Gobierno Vasco, 17 pp. 
I.P.C.C. (2014). Climate Change 2014. Mitigation of Climate Change. Contribution of Working Group III to the Fifth Assessment Report of the Intergovernmental Panel on Climate Change. Cambridge University Press, Cambridge, 1435 pp.

Kim, H.M.; Rutqvist, J.; Ryu, D.W.; Choi, B.H.; Sunwoo, C. \& Song, W.K. (2012). Exploring the concept of compressed air energy storage (CAES) in lined rock caverns at shallow depth: a modelling study of air tightness and energy balance. Applied Energy, 92: 653-667. https://doi.org/10.1016/j.apenergy.2011. 07.013

Kyriakopoulos, G.L. \& Arabatzis, G. (2016) Electrical energy storage systems in electricity generation: Energy policies, innovative technologies, and regulatory regimes. Renewable and Sustainable Energy Reviews. 56: 1044-1067 https://doi.org/10.1016/j. rser.2015.12.046

Heijdra, J.J. \& Prij, J. (1992). Convergence measurements in a $300 \mathrm{~m}$ deep borehole in rock salt. Netherlands Energy Research Foundation ECN, 24 pp.

Hoek, E. \& Brown, E.T. (1980). Underground excavations in rock. The Institution of Mining and Metallurgy, London, 527 pp.

Hoek, E. (1990). Estimating Mohr-Coulomb friction and cohesion values from the Hoek-Brown failure criterion. International Journal of Rock Mechanics and Mining Sciences \& Geomechanics Abstracts, 12(3): 227-229. https://doi.org/10.1016/0148-9062(90)94333-O

Ibrahim, H.; Ilinca, A. \& Perron, J. (2008) Energy Storage Systems - characteristics and comparisons. Renewable and sustainable energy reviews. 12: 1221-1250. https://doi.org/10.1016/j.rser.2007.01.023

King, M.S. (1983). Static and dynamic elastic properties of rocks from the Canadian Shield. International Journal of Rock Mechanics and Mining Sciences \& Geomechanics Abstracts, 20(5): 237-241. https://doi. org/10.1016/0148-9062(83)90004-9

Lanaja J.M. \& Navarro, A. (1987) Contribución de la exploración petrolífera al conocimiento de la geología de España. IGME, 465 pp.

Liang, G.C.; Huang, X.; Peng X.Y.; Tian, Y. \& Yu, Y.H. (2016) Investigation on the cavity evolution of underground salt cavern gas storage. Journal of Natural Gas Science and Engineering. 33: 118-134. https:// doi.org/10.1016/j.jngse.2016.05.018
Llamas, B.; Castañeda, M.C.; Laín, C. \& Pous, J. (2017a). Multi-criteria algorithm-based methodology used to select suitable domes for compressed air Energy storage. International Journal of Energy Research. 41 (14): 2108-2120. https://doi.org/10.1002/er.3771

Llamas, B.; Castañeda, M.C.; Laín, C. \& Pous, J. (2017b). Study of the Basque-Cantabrian basin as a suitable region for the implementation of an energy storage system based on compressed air underground storage (CAES). Environmental Earth Sciences. 76: 204. https://doi.org/10.1007/s12665-017-6515-y

Llamas, B.; Laín, C.; Castañeda, M.C. \& Pous, J. (2018). Mini-CAES as a reliable and novel approach to store renewable energy in salt domes. Energy, 144(1): 482-489.

Liang G-c, Huang X, Peng X-y, Tian Y, Yu Y-h. (2016) Investigation on the cavity evolution of underground salt cavern gas storage. Journal of Natural Gas Science and Engineering, 33: 118-134. https://doi. org/10.1016/j.jngse.2016.05.018

Lund, H. \& Salgi, G. (2009) The role of compressed air energy storage (CAES) in future sustainable energy systems. Energy Conversion and Management. 50: 1172-1179. https://doi.org/10.1016/j.enconman.2009. 01.032

Luo, X.I.N.G. \& Wang, J. (2013). Overview of current development on compressed air energy storage. EERA Technical Report, School of Engineering, University of Warwick, Coventry, 38 pp.

Luo, X.; Wang, J.; Dooner, M. \& Clarke, J. (2015). Overview of current development in electrical energy storage technologies and the application potential in power system operation. Applied Energy, 137: 511-536. https://doi.org/10.1016/j.apenergy.2014.09.081

Madlener, R. \& Latz, J. (2013). Economics of centralized and decentralized compressed air energy storage for enhanced grid integration of wind power. Applied Energy. 101: 299-309. https://doi.org/10.1016/j. apenergy.2011.09.033

McCartney, J.S.; Sanchez, M. \& Tomac, I. (2016). Energy geotechnics: advances in subsurface energy recovery, storage, exchange, and waste management. Computers and Geotechnics, 75: 244-256. https://doi. org/10.1016/j.compgeo.2016.01.002

Vera, J.A. (2010) Geología de España. Instituto Geológico y Minero de España, Madrid, 884 pp. 